\title{
INTERGALACTIC MATTER AND RADIATION
}

\author{
G. R. BURBIDGE \\ University of California, San Diego, Calif., U.S.A. and \\ Institute of Theoretical Astronomy, Cambridge, Great Britain
}

\begin{abstract}
This paper presents a critical discussion of our present knowledge of intergalactic matter and radiation as it relates to cosmology. While there have been some observational successes and much theoretical speculation, studies of intergalactic matter and radiation have still not allowed us to draw certain conclusions about the origin and evolution of the universe.
\end{abstract}

\section{Introduction}

One of the most important quantities which we need to detect and understand from the point of observational cosmology is the total amount of matter and radiation in the universe. We are able to detect comparatively easily the discrete sources which emit electromagnetic radiation, but it would be very rash to assume that these are all that are present in the universe.

Other components which may be present are:

(a) Mass condensations which are not emitting enough radiation to be detectable;

(b) Smooth distributions of matter;

(c) Smooth electromagnetic radiation fields;

(d) Gravitational radiation;

(e) High energy particle fluxes;

(f) Neutrinos.

Knowledge of the relative importance of these different components can certainly be expected to increase our understanding (or, more appropriately, decrease our ignorance by a small amount) concerning the formation and evolution of galaxies and other condensed objects. If we could make a direct estimate of the total massenergy density of all of these components, $\varrho_{c}$, this could be directly used to restrict the possible cosmological models, the point being that, in the Friedman models and in the steady-state models, $\varrho_{c}$ is directly related to the other cosmological parameters $H_{0}, \Lambda, q_{0}$ by the relations

$$
\begin{array}{rlrl}
\Lambda+3 H_{0}^{2} q_{0} & =4 \pi G\left(\varrho_{0}+\frac{3 p}{c^{2}}\right) & & (\text { Friedman models }) \\
\varrho_{s s} & =\frac{3 H_{0}^{2}}{8 \pi G} & (\text { steady state }) .
\end{array}
$$

It is customary to start from the fact that the mass density of matter condensed in bright galaxies is about $3 \times 10^{-31} \mathrm{~g} \mathrm{~cm}^{-3}$, assuming a Hubble constant of $75 \mathrm{~km} \mathrm{~s}^{-1} \mathrm{Mpc}^{-1}$. This value is obtained by estimating the number density of galaxies from the counts 
and by assigning average masses from individual mass determination for a sample of nearby galaxies (cf. the review by Burbidge and Burbidge, 1970) or by using the luminosities and mass-to-light ratios obtained by direct determinations from nearby galaxies. The number that we use is usually attributed to Oort (1958). However, it is interesting to point out that it can be roughly derived from early work by Hubble (1936), if we simply adjust the Hubble constant (from $550 \mathrm{~km} \mathrm{~s}^{-1} \mathrm{Mpc}^{-1}$, used by him, to $75 \mathrm{~km} \mathrm{~s}^{-1} \mathrm{Mpc}^{-1}$ ) and take account of the uncertainties in the mass determinations available at that time. (Since the mass density $=n_{g} \bar{M}$ and since $n_{g} \propto H_{0}^{3}$ and $\left.\bar{M} \propto H_{0}^{-1}, \varrho_{g} \propto H_{0}^{2}\right)$. Hubble obtained a value ranging between $10^{-30}$ and $10^{-28} \mathrm{~g} \mathrm{~cm}^{-3}$. If we take a mean $=10^{-29} \mathrm{~g} \mathrm{~cm}^{-3}$, we find that the adjustment due to the change in the Hubble constant gives a value of $2 \times 10^{-31} \mathrm{~g} \mathrm{~cm}^{-3}$, very close to the value given by Oort and others. Abell (1965) has given upper and lower limits which are rather higher and which have been quoted by some. However, these numbers are somewhat misleading in that they are based on the masses of clusters and the upper limits are the mean densities in clusters. Moreover, the masses of the clusters are obtained from the virial theorem. Now it is well known that the application of this method - using known masses and mass-to-light ratios - leads to the conclusion either that the clusters are not bound, or that much hidden mass (not in the form of normal galaxies) is present. Thus Abell's estimates are either too high, or they already include a significant mass contribution which is not galaxies. The so-called 'critical' density which is such that a Friedman universe will expand to a finite maximum radius and then contract (the oscillating model) is obtained when $\Lambda=0, q_{0}=\frac{1}{2}$. In this case we find from Equations (1) and (2)

$$
\varrho_{c}=\varrho_{s s}=3 H_{0}^{2} / 8 \pi G=1.1 \times 10^{-29} \mathrm{~g} \mathrm{~cm}^{-3} .
$$

Since the density in the form of normal galaxies is much less than this, we have the problem of the so-called 'missing mass'. Various approaches have been taken to it.

Some have argued that it is most likely that the universe is closed. Thus a large part of the mass energy is not in ordinary galaxies and must be contained in other components. Others have taken the frequently quoted value of $q_{0} \sim+1$ which has been obtained from analyses of the redshift-apparent magnitude diagram for galaxies by Sandage and his colleagues, at face value, to argue that much hitherto undiscovered mass must be present. Also, those who accept the steady-state cosmology require that the bulk of the mass is not in the form of normal galaxies.

On the other side, the view has been expressed by some theoretical cosmologists that the mass in galaxies is substantially all that is present, and that therefore the universe is open since $\varrho_{g} \ll \varrho_{c}$.

Regardless of the theoretical arguments for and against the possibility that missing mass may be present, we shall take the view that detection of intergalactic matter and radiation is of primary importance, observations of a null character which will loom large in this discussion are of lesser importance, and that theoretical analyses of what turns out to be a hypothetical intergalactic medium are, at present, only of minor interest. 


\section{Detection of Discrete Intergalactic Objects by Observation of Photons Emitted by Them}

\section{A. INTERGALACTIC BRIDGES}

The most obvious types of intergalactic matter that can clearly be seen are the bridges and extended filaments between galaxies which have been described by Zwicky (1957), and by Vorontsov-Velyaminov (1959) and Arp (1966) in their catalogues of peculiar galaxies. Apart from stating that such bridges can be seen associated with many galaxies, we have little or no detailed information on the composition of such bridges, or the amount of matter present. In practically no case have accurate measurements of the spectrum and energy distribution of such systems been made, largely because of the low surface brightness involved. Thus we do not know whether they are composed of stars, or of gas, or of a combination of both. Indeed, the possibility that some part of the optical luminosity is due to non-thermal radiation mechanisms of the synchrotron type, though it is very unlikely, cannot be excluded.

And, of course, the fundamental theoretical question as to whether such matter originated in the galaxies and has been drawn out by tidal interactions, or ejected by explosive processes, or whether it is matter which is condensing onto the galaxies, is also unanswered. However, the likelihood in most cases is that it is matter which has been ejected.

\section{B. INTERGALACTIC MATTER IN CLUSTERS OF GALAXIES}

Studies of the two richest comparatively nearby clusters, the Virgo Cluster and the Coma Cluster, have suggested that there is low-luminosity matter between the galaxies in the central region. In his book Zwicky (1957, p. 48) stated that, using the 48-in. Palomar Schmidt, he had discovered photographically an extended mass of low surface brightness material spread throughout the central part of the Coma Cluster. The very difficult problem involved in measuring such a background has been discussed by Abell (1965). Recent photoelectric investigations by de Vaucouleurs and de Vaucouleurs (1970) give a peak intergalactic brightness of $26.2 \mathrm{mag} . \mathrm{s}^{-2}$, and they state that the total magnitude of the background amounts to about $40 \%$ of the integrated luminosity of the galaxies as they are normally defined. They point out that the background can be largely accounted for by the overlapping coronas of the two supergiant galaxies in the cluster, NGC 4874 and NGC 4889, and a few others.

Many years ago Baum (1955) attempted to measure the surface brightness of M87, one of the brightest galaxies in the Virgo Cluster, very far from its center, and found no deviation from the Hubble $1 / r^{2}$ law however far he went. More recent observations by Arp and Bertola (1969) and by de Vaucouleurs (1969) showed the presence of a very extended exponential corona of very low surface brightness and low luminosity gradient which could be measured out to some $100-150 \mathrm{kpc}$ from the nucleus of the galaxy.

At present it appears therefore that the luminous material is likely to be extended overlapping coronas of giant elliptical galaxies, and not a uniform, but dynamically 
separate background, extending through the whole cluster. The observers have assumed without proof that this luminous material is made up of stars; this is plausible, but not necessarily correct. The absolute magnitudes of the galaxies are increased significantly, but there is no evidence that there is sufficient luminous material in these clusters to resolve the problem associated with the stability of these clusters.

\section{LOW LUMINOSITY SYSTEMS}

The discoveries made in the last few years have shown that there is a wide population of condensed objects which are not counted as normal galaxies. In various places in the literature Zwicky has stressed the possible importance of such objects, and the fact that they should be looked for, but little attention was paid to this suggestion until observations made with quite different ideas in mind led to the discovery of new classes of objects. There are various pieces of evidence that must be mentioned.

(i) Hubble, Baade, and others believed throughout their professional lives that the luminosity function for galaxies has a roughly gaussian form. On the other hand, Zwicky continuously claimed that the luminosity function increases monotonically with decreasing luminosity. If it is assumed that the mass-to-light ratio for all galaxies is the same, i.e. that they contain the same stellar populations, then the mass function of galaxies will have the same form. One of the problems that we face is that the mass-to-light ratios are clearly not the same for all stellar systems; in fact there is some evidence that the mass-to-light ratio is itself an increasing function of the mass. However, it is now clear that the luminosity function for galaxies, as far as it has been taken in studies of nearby clusters and galaxies in the vicinity of our own Galaxy, is of the form proposed by Zwicky. Abell (1965) gives, for example, the number of galaxies with luminosities greater than $L$, for the Coma Cluster,

$$
N(>L) \propto L^{-0.75} \text {. }
$$

It is therefore clear that the total number of stellar systems is increasing with decreasing luminosity, and it may be asked what bearing this has on the estimates of the mass density in the universe.

In his calculations Oort (1958) used the luminosity function obtained by Holmberg for the Virgo Cluster. However, he did take into account the possibility that the luminosity function continued indefinitely to lower values according to the suggestions of Zwicky, but concluded that the contribution to the total mass from lower luminosity systems (fainter than -17) was less than $25 \%$. It is clear that no significant increase can be obtained unless the differential mass distribution (or luminosity distribution if $M \propto L$ with the same constant of proportionality through the whole range) is of the form $\mathrm{d} M / M^{\alpha}$, with $\alpha \geqslant 1$. There is no evidence that for the normal stellar systems the mass function is anything like as steep as this. However, we really do not know how steep it is. A large population of what appear to be dwarf galaxies has been detected in the Virgo Cluster (Rubin et al., 1967). Also, a number of intergalactic globular clusters have been detected essentially by accident, and no one has any real idea of the space distribution of such objects. 
There is also now evidence of the existence of what Zwicky termed compact galaxies, which he defined as those which can just be distinguished from stars on plates taken with the 48-in. Schmidt telescope and which have diameters of $2^{\prime \prime}$ to $5^{\prime \prime}$. Earlier he had defined them in a slightly different way. The spectroscopic investigations of Sargent (1970) show that they are a heterogeneous collection. However, it appears that many have the following properties.

(1) They are compact in the sense that their surface brightnesses are greater than those of the classical ellipticals: i.e., they have smaller effective radii, but a comparable range of absolute magnitudes.

(2) The line spectra are often similar to those of normal ellipticals so that, as a first approximation, it can be assumed that the velocity dispersions of the stars are similar.

If the velocity dispersions are similar, then if the structures are homologous with the normal ellipticals, $M \propto R_{\text {eff }}$ and consequently it might be supposed that they have smaller masses, and thus smaller mass-to-light ratios than normal ellipticals. Thus, while they have not previously been included in the estimates of the number of galaxies which go into the determination of $\varrho_{g}$, unless their space density is exceedingly high compared with normal galaxies, they are not likely to increase the estimates of $\varrho_{g}$ significantly.

\section{STAR-LIKE OBJECTS WITH NON-THERMAL SPECTRA}

One of the most remarkable developments in the last few years has been the realization that there is a large population of star-like extragalactic objects which radiate a wide spectrum ranging from radio to ultraviolet wavelengths, and possibly into the $\mathrm{X}$-ray region, by non-thermal processes. Since it is now known that the nuclei of many galaxies also exhibit the same behaviour, one possibility is that the fainter star-like objects are indeed the nuclei of normal galaxies which become so bright and energetic at some stages that they overwhelm the stellar radiation from the galaxies. This is indeed what many people believe, and if they are correct the discovery of the QSOs does not mean that there is a new class of object to be taken into account. On the other hand, these objects may not have originated in galaxies at all, and in this case they make a significant contribution to $\varrho_{g}$. The number has been estimated by Sandage and Luyten (1968) to amount to some 200 per square deg down to about $22^{m}$, and there is some indication that there is a maximum in the distribution in apparent magnitude between about $18^{m}$ and $19^{m}$. Thus there may be about $10^{7}$ over the whole sky. The maximum in the number-apparent magnitude distribution, if real, and the redshift distribution would mean that the universe becomes opaque at an epoch corresponding to a redshift somewhat greater than 2 , and that there is an evolutionary effect which is proportional to $(1+z)^{\alpha}$, with $\alpha \simeq 6$, with a cutoff beyond $\sim 2.4$. Alternatively some attribute the effect purely to evolution as a function of epoch. All of this, if the redshifts are cosmological in origin. These numbers then give a mean space density $\sim 10^{-78} \mathrm{~cm}^{-3}$. However, it appears that the lifetimes of these objects in their luminous phases are only $\sim 10^{6}-10^{7} \mathrm{yr}$ so that the space density of massive objects which give rise to them over $\sim 10^{10} \mathrm{yr}$ will be about $10^{-77} \times 10^{10} /\left(10^{6}-10^{7}\right)$ or about $10^{-73}$ 
$10^{-74} \mathrm{~cm}^{-3}$. If the average masses are $10^{10}-10^{11} M_{\odot}$, they will make a contribution $\varrho_{Q} \simeq 10^{-29}-10^{-31} \mathrm{~g} \mathrm{~cm}^{-3}$. Of course the values that have been put in here for the masses are quite uncertain, but this elementary calculation shows that such objects might make a significant contribution to the mass density.

If the redshifts are not cosmological, then the possibilities are that we are looking out no further than redshifts of $\sim 0.2$ or that the objects are in a 'local' cloud whose outer boundary is no greater than about $100 \mathrm{Mpc}$, and that the next cloud is far enough away so that the brightest objects have apparent brightnesses much fainter than $22^{m}$. In either case the space density will be much higher than is the case if they are at cosmological distances, but since the objects are much less energetic than is the case if they are at cosmological distances, they may have quite modest masses and thus not make a large addition to $\varrho_{g}$.

Thus, while we now know that compact extragalactic systems may be very common, we have so far found no way of estimating with any precision what their masses are, and hence we do not know what contribution they may make to the mass energy balance in the universe. Some attempts have been made to estimate the total amount of luminous matter in the universe from the observed light of the night sky (Peebles and Partridge, 1967; Roach and Smith, 1968). Peebles and Partridge showed that, if the matter has $M / L=20$, the upper limit to the mass is a factor of four times lower than the critical density. The result depends sensitively on the actual value of the sky brightness due to extragalactic sources as distinct from that arising by airglow, zodiacal light, and galactic starlight. Since the value used to set the limit is much smaller (by a factor of 10 ) than the contribution attributed to zodiacal light, the method is clearly rather uncertain.

\section{Intergalactic Gas}

Whatever type of universe we live in, it is generally agreed that some intergalactic gas is likely to be present. In the picture of a universe starting in a big-bang, it is argued that not all of the matter will condense into galaxies, while in the older version of the steady-state universe, it is argued that baryons and leptons are being uniformly created in space so that much of the matter may be present in the form of intergalactic gas.

With these ideas as a background, many theoretical papers have been written, particularly in the framework of evolving cosmologies, on the evolution of this hypothetical intergalactic gas. We shall discuss these later. However, more important are the attempts to find some evidence for the presence of intergalactic gas.

\section{A. RADIO OBSERVATIONS}

The first attempts were made using the $21-\mathrm{cm}$ line transition in neutral atomic hydrogen. Attempts were made to detect intergalactic hydrogen in both absorption and emission. Field (1962) attempted to detect intergalactic hydrogen by looking for absorption in the spectrum of Cygnus A. He was not able to detect any absorption, and concluded in a thorough discussion that: 
(a) The intergalactic medium may be hot $\left(T_{K}>10^{4} \mathrm{~K}\right)$.

(b) The medium may be cool with $T_{K}<10^{4} \mathrm{~K}$ and yet considerably ionized (greater than $40 \%$ ) by ultraviolet radiation or charged particles.

(c) The medium may be cool $\left(<10^{4} \mathrm{~K}\right)$ and very little ionized. In this case the density must be $<10^{-29} \mathrm{~g} \mathrm{~cm}^{-3}$, significantly below the critical value.

Davies and Jennison (1964) carried out a similar investigation, and also obtained a null result with a somewhat lower limit. Goldstein (1963) attempted to detect $21-\mathrm{cm}$ emission from intergalactic gas and again obtained a null result, suggesting that $\varrho_{g}<10^{-29} \mathrm{~g} \mathrm{~cm}^{-3}$. However, Koehler and Robinson (1966) claimed to have detected neutral hydrogen, both in the Virgo Cluster and in intergalactic space in the direction of the source Fornax A. Both measurements were made by the absorption method. Neither measurement can be confirmed when higher sensitivity is used. Penzias and Scott (1968) carried out a further study looking for 21-cm absorption in the spectrum of Cygnus A. Their sensitivity was considerably better than any used previously, and they still obtained a null result. Penzias and Wilson (1969) attempted to detect intergalactic emission at $21-\mathrm{cm}$, and again found a null result leading them to the conclusion that:

$$
\varrho_{g}<5 \times 10^{-30} \mathrm{~g} \mathrm{~cm}^{-3}, \text { or } n_{g}<3 \times 10^{-6} \text { atoms cm }{ }^{-3},
$$

provided that the spin temperature of the atoms is large compared with the background. But their null result from the absorption led to the conclusion that

$$
n_{g} / T_{s}<2.3 \times 10^{-8} \text { atoms } \mathrm{cm}^{-3} \text {. }
$$

Thus any spin temperature close enough to the temperature of the microwave background to affect the validity of the emission result will lead immediately to an even smaller upper limit based on the absorption result.

The work of Koehler and Robinson on absorption in the Virgo Cluster has also been shown to be in doubt. Allen (1969) has shown that there is no detectable absorption in the radio spectrum of $\mathrm{M} 87$, and hence he can find no evidence for any neutral hydrogen in the Virgo Cluster or between our Galaxy and M87. He has concluded that the density of intergalactic hydrogen cannot be greater than $10^{-30} \mathrm{~g} \mathrm{~cm}^{-3}$ unless it is more than $70 \%$ ionized, or unless it is concentrated into clouds, very few of which lie along the line of sight.

Thus the conclusion from all of the radio work so far is that there is no evidence at all for the presence of diffuse intergalactic gas.

\section{B. HIGH LATITUDE CLOUDS OF HYDROGEN DISCOVERED FROM 21-CM LINE STUDIES}

The Leiden group have discovered, in surveys at high galactic latitudes, clouds of neutral atomic hydrogen which appear to be falling into the Galaxy with velocities $\sim 100 \mathrm{~km} \mathrm{~s}^{-1}$ (Oort, 1966, 1969; Hulsbosch, 1968). This may be intergalactic gas which is being swept up by the Galaxy, as Oort believes, more distant clouds, or gas which was originally ejected from the center or from vast explosions in the plane. 
If the clouds seen are due to the effects of infalling material, it is not possible to obtain directly the density of intergalactic matter because, for dynamical reasons, it is necessary to suppose that this matter must have interacted with halo material which in turn has been ejected from the plane of the Galaxy. Oort (1970) states that van Leer has estimated the intergalactic density to be about $3 \times 10^{-28} \mathrm{~g} \mathrm{~cm}^{-3}$ immediately outside the Galaxy. While this density is considerably greater than $\varrho_{c}$, it is representative of an intragalactic density in the local group of galaxies rather than the density of gas between groups and clusters of galaxies, and much of the gas is that which was originally thrown up into the halo from the disk.

If this model is correct it is necessary to reconcile the result with the null result concerning hydrogen in the Virgo Cluster.

\section{OPTICAL OBSERVATIONS}

Little attention was paid to the possibility of detection of intergalactic gas by optical methods until the advent of space astronomy and the discovery of the QSOs. It was realized that a very powerful test was available if one could observe extragalactic objects in the region of the Ly- $\alpha$ wavelength. For absorption of Ly- $\alpha$ radiation by intergalactic hydrogen lying in the light path between the object and the observer may be expected to give rise to an absorption trough in the continuum which extends from immediately to the blue of the Ly- $\alpha$ emission radiated by the source, assumed to have a non-zero redshift, to close to the rest wavelength of $\mathrm{Ly}-\alpha$.

To observe the region of $\mathrm{Ly}-\alpha$ at all it is necessary either to observe from above the Earth's atmosphere, and observe objects at redshifts $\left(\geqslant 1000 \mathrm{~km} \mathrm{~s}^{-1}\right)$ great enough to avoid the effects of the interstellar hydrogen in and close to our Galaxy, or to observe objects with redshifts so large $(z \geqslant 2)$ that Ly- $\alpha$ is moved into the visible part of the spectrum. The first method was discussed by Burbidge (1964), but still it has not been applied since no extragalactic objects fulfilling these requirements have yet been observed from space.

With the discovery of the first quasi-stellar object with a redshift $\sim 2,3 \mathrm{C} \mathrm{9}$, by Schmidt in 1965, Gunn and Peterson (1965) and Scheuer (1965) proposed that the second method be applied, and Gunn and Peterson analysed the first spectra of 3C 9. They concluded that they could see the beginning of a smooth absorption trough to the violet of the Ly- $\alpha$ emission line and deduced from this that the density of intergalactic hydrogen comparatively close to $3 \mathrm{C} 9$ was $\varrho_{g}=1 \times 10^{-34} \mathrm{~g} \mathrm{~cm}^{-3}$. However, studies of more spectra of 3C 9 and of many other QSOs which are now known with redshifts such that the continuum level around Ly- $\alpha$ can be studied have led to the conclusion that there is no measurable absorption trough present. From this we are led to the conclusion (Burbidge and Burbidge, 1967):

(a) Either that the QSOs are not at cosmological distances; or

(b) that $\varrho_{g} \leqslant 10^{-35} \mathrm{~g} \mathrm{~cm}^{-3}$, if the diffuse gas is largely in the form of neutral atomic hydrogen.

Most students of the intergalactic medium have chosen to ignore (a) and concentrated on (b). Since we are discussing the intergalactic medium we shall not digress 
on (a) further here, though it is of importance if we are interested in the general problem of the nature of the QSOs.

The next developments in optical investigations of intergalactic gas came after the suggestion by Bahcall and Salpeter (1966) and Shklovsky (1964) that intergalactic gas might be condensed into discrete clouds so that it might be possible to detect it by looking for discrete absorption lines in the spectra of QSOs if they are at cosmological distances. The absorption line redshift(s) $z_{\text {abs }}$ would be expected to be less than $z_{\mathrm{em}}$. The method is entirely analogous to that used to study interstellar clouds in the Galaxy by looking for interstellar absorption lines in stellar spectra. Very soon after these suggestions were made, the first QSO (3C 191) which had a number of strong absorption lines in its spectrum was discovered (Burbidge et al., 1966; Stockton and Lynds, 1966). However, in this object it was found that $z_{\mathrm{abs}} \simeq z_{\mathrm{em}}=1.95$, so that it was immediately clear that the absorption in this object is associated with the QSO and does not arise in an intervening medium. Following this discovery, many QSOs with absorption lines were discovered, but in the majority of cases $z_{\text {abs }} \approx z_{\text {em }}$ and in some cases $z_{\text {abs }}$ is slightly greater that $z_{\text {em }}$. However, in 3 or 4 cases out of about 20 (see Burbidge and Burbidge, 1969a) several absorption redshifts are present, some very much smaller than the emission redshift of the QSO. This is the kind of effect that could be expected if the absorption were due to an intervening medium.

In the theoretical literature, the impression has been given by continuous reference to these objects, particularly by Bahcall and his theoretical associates, that such QSOs are typical of the majority and that the absorption is likely to be of intergalactic origin (cf. a particularly blatant example of this by Silk, 1970). Attempts to calculate the probability of finding a number of discrete absorption redshifts by intervening objects, dead galaxies (Bahcall and Peebles, 1969), halos around galaxies (Bahcall and Spitzer, 1969), etc., assuming that multiple absorption redshifts are the rule, rather than the exception, have all obscured until recently the main observational results for all of the absorption line material taken together, namely that $\left|z_{\mathrm{em}}-z_{\mathrm{abs}}\right|$ is in the majority of cases very small. A histogram of redshift differences $\left(z_{\mathrm{em}}-z_{\text {abs }}\right)$ is shown by E. M. Burbidge (1970). Also, spectroscopic arguments and statistical arguments suggest very strongly that the lines are all formed in the QSOs and that the different redshifts are intrinsic to the objects themselves. Recently theoretical attempts to explain these multiple redshifts on this basis have been made by Shklovsky (1969) and by Rees (1970). From all of these investigations the conclusion at present is that there is no compelling evidence at all for the presence of any absorption of intergalactic origin in the QSOs. This may again be taken as evidence that the QSOs are not at cosmological distances, or that intergalactic matter in the form of cool clouds is absent.

As well as looking for Ly- $\alpha$ absorption from intergalactic gas, attempts have been made to detect Ly- $\alpha$ emission, which will be smeared into a continuum by the redshift effect, and continuum ultraviolet radiation. Attempts to study the ultraviolet radiation from an intergalactic medium have been made by Kurt and Sunyaev (1967a, 1967b, 1970). They have shown that the measurements in the range $1225 \AA-1340 \AA$ set an 
upper limit of $\varrho_{g}<5 \varrho_{c}$ for any assumed temperature of intergalactic gas, and $\varrho_{g}<\varrho_{c}$ for the narrower interval $3 \times 10^{4}>T>10^{4} \mathrm{~K}$.

\section{X-RAY OBSERVATIONS}

We consider now attempts to detect a hot intergalactic gas. An early theoretical proposal was that of Gold and Hoyle (1959) who suggested that, in a steady-state universe, matter might be created as neutrons which would then decay, giving rise to a very high temperature gas. However, when the first observations of a diffuse X-ray background flux in the energy range $1-10 \mathrm{keV}$ were made, it was possible to show (Gould and Burbidge, 1963; Field and Henry, 1964) that such a hot universe with a density required by steady-state theory (equal to the critical density) could be ruled out, as it would give rise to an X-ray background flux much larger than that already observed. For gas at critical density these results already required that $T \leqslant 5 \times 10^{6} \mathrm{~K}$. We now have many observations of the background X-ray flux, and the form and intensity of the spectrum for energies $\gtrsim 1 \mathrm{keV}$ indicate that it does not arise as bremsstrahlung from a hot intergalactic gas. However, observations made in the soft X-ray region ( $0.25 \mathrm{keV}$ ) (Henry et al., 1968; Bowyer et al., 1968; Bunner et al., 1969) have shown that a flux is being emitted which Henry et al. originally claimed was due to a hot intergalactic plasma with a temperature of about $0.8 \times 10^{6} \mathrm{~K}$ and with $\varrho=\varrho_{c}$. However, the interpretation of the observations is complicated by the fact that it is necessary to correct the raw data for absorption of these soft X-rays in the vicinity of our own Galaxy. The amount of this correction has been much in dispute. The strength of the argument that this radiation is being generated by a process different from that of the remainder of the background depends, to a considerable extent, on how far above the extrapolation of a power law spectrum the corrected soft X-ray measurements lie. The most recent observations (Bunner et al., 1969) still show that there is great uncertainty, but they have measurements over a wide enough spectral range so that they conclude that either a steady-state density model with a temperature $\sim 3 \times 10^{6} \mathrm{~K}$ (as discussed by Field and Henry) is compatible with the data, or an evolving model with $T \sim 10^{6} \mathrm{~K}$ and $\varrho_{g} \sim \varrho_{c} / 6$. It is also clear, as they and others have stressed, that discrete sources of soft X-rays, either in the halo of our Galaxy or on the extragalactic scale, might also explain the data.

Sunyaev (1969) has pointed out that limits to the density and temperature of hot intergalactic gas may be set by considering its ionizing effects on neutral hydrogen in the outer parts of galaxies. He concluded that the observed distribution of neutral hydrogen around galaxies such as M31 meant that a uniform intergalactic plasma with $T>10^{5} \mathrm{~K}$ and the critical density could not be present. However, in a further analysis Felten and Bergeron (1969) demonstrated that the presence of a hot intergalactic plasma at the critical density is compatible with the observations, even at the higher flux level originally used by Henry et al. (1968).

From the X-ray observations, it must be concluded that there is only preliminary tentative evidence for the existence of a hot intergalactic plasma. If it is responsible for the soft X-ray flux observed, rather than a class of discrete sources, it appears that 
the density of this plasma in a Friedman universe is only $\sim 10^{-30} \mathrm{~g} \mathrm{~cm}^{-3}$, far less than $\varrho_{c}$.

\section{E. OBSERVATIONS CONCERNED WITH INTERGALACTIC GAS AT $z \geqslant 2$}

QSOs have been observed with redshifts up to 2.9 , the vast majority having values less than about 2.2. Of about 200 redshifts measured only 3 are larger than 2.2 . The cutoff in the redshifts above about 2.2 is generally accepted to be a real effect (Burbidge and Burbidge, 1969b). To explain this result on the cosmological hypothesis, it is necessary to explain this in one of three ways;

(1) The steeply increasing density function of QSOs $\varrho(z) \propto(1+z)^{6}$ must rapidly flatten off beyond $z \sim 2.2$ and this must be due to the physical conditions associated with the formation or appearance of QSOs.

(2) The distribution of redshifts might be explained in the context of a Lemaitre universe with a 'coasting' time near $z \simeq 2$.

(3) Intergalactic absorption may be responsible for the cutoff. Rees (1969) explored (3), and concluded that the explanation could be made plausible within the framework of theoretical views concerning the history of the hypothetical intergalactic gas (to be discussed later) with the absorption being due to atomic hydrogen, the gas being less highly ionized at early epochs than when $z \lesssim 2$.

The discovery of a very large redshift in 4C 05.34 (Lynds and Wills, 1970) of $z=2.88$ leads now to some complications. It is necessary to postulate that the absorption is not uniform so that, in some directions, it is possible to look back much further than in others. Also, the object does not have broad absorption bands arising from the Lyman series to the violet of Ly- $\alpha$ emission in the object.

\section{F. OPTICAL EVIDENCE FOR INTERGALACTIC DUST}

There is some tentative evidence for intergalactic dust. Some galaxies appear to have outer regions partially obscured by dust which may have an intergalactic origin. Also, Zwicky (1957), using statistical arguments concerned with the apparent distribution of galaxies, has argued that intergalactic dust clouds are fairly plentiful. However, in other analyses concerned with galaxy distributions (Neyman et al., 1952; Karachentsev, 1966; Yu and Peebles, 1969) attempts are made to study first and second order clustering without invoking the presence of dust.

\section{Conclusions Regarding the Presence of Large Amounts of Condensed or Diffuse Matter in The Universe}

Despite all the attempts to detect intergalactic matter, the present situation is that almost nothing has been found, and certainly nothing which would give us any observational reason to believe that the critical density, equal to the steady-state density of matter, is present. There is very tentative evidence for some hot gas with $\varrho<\varrho_{c}$, some evidence that there is some cold hydrogen in the vicinity of our Galaxy, 
though this is not truly intergalactic matter, and the discovery of various types of compact luminous objects leads naturally to the conclusion that there must be a population of condensed objects which have so far not been included when the mass density of galaxies was determined. All of the other observations are null observations. The simplest interpretation of these results is that we live in an open universe of Friedman type (Zeldovich and Sunyaev, 1969), or that we live in a closed universe, or a steady-state universe, in which the bulk of the mass is in the form of dark condensed objects with very high $M / L$ ratios or in gravitational radiation.

Theoretical views concerned with the evolution of matter in the universe will be discussed later, but now we turn to the observed properties of radiation in the universe.

\section{Electromagnetic Radiation in the Universe}

There are four distinct types of background radiation which are known to pervade intergalactic space. They are the microwave background radiation (prematurely called 'black-body' radiation by many, and 'relict radiation' also assuming that its origin is understood, by the Russian cosmologists); star-light, non-thermal radio frequency radiation, and X-ray background radiation. We discuss in turn the observed properties of these radiation fields and their possible origins.

\section{A. MICROWAVE BACKGROUND RADIATION}

Since black-body radiation at a temperature of a few degrees Kelvin was predicted to be present at this epoch if the universe has expanded from a dense initial state, the discovery of a highly isotropic radiation field has been widely interpreted as confirmation of the theoretical prediction. The observed flux of radiation with wavelengths from about $20 \mathrm{~cm}$ to a few millimeters fits quite well on a black-body curve with a temperature of about $2.7 \mathrm{~K}$. The energy density of the radiation is then about $6 \times 10^{-13} \mathrm{erg} \mathrm{cm}{ }^{-3}$, corresponding to a mass density of $\sim 7 \times 10^{-34} \mathrm{~g} \mathrm{~cm}^{-3}$. If the black-body form is confirmed, this will be the strongest evidence in favor of an evolving universe. However, it is not yet certain whether or not the spectrum is really of black-body form, since the wavelength range directly observed, and not in dispute, is the Rayleigh-Jeans part of the curve where $I(v) \propto v^{2}$.

The observations which have been made either directly or indirectly close to, and just beyond, the peak of the black-body curve are in conflict unless it is argued that some very strong line radiations are present. The indirect observation of the radiation field in the galactic plane which is exciting the $\mathrm{CN}, \mathrm{CH}$, and $\mathrm{CH}^{+}$interstellar molecules suggests that the temperature is less than $\sim 8 \mathrm{~K}$ at $9.36 \mathrm{~mm}$, less than $5 \mathrm{~K}$ at $0.56 \mathrm{~mm}$, less than $\sim 4 \mathrm{~K}$ at $1.3 \mathrm{~mm}$, and it appears to be about $2.8 \mathrm{~K}$ at $2.64 \mathrm{~mm}$ (Bortolot et al., 1969). However, direct observation from rockets and, more recently, a balloon above the Earth's atmosphere at wavelengths in the range $0.4-1.3 \mathrm{~mm}$ gives a flux which corresponds to a black-body temperature of about 7K (Shivanandan et al., 1968; Houck and Harwit, 1969; Muelhner and Weiss, 1970). It is possible that the radiation detected by these observers is concentrated in a line which could be 
produced in a geocorona, in the Galaxy, or have a cosmological origin. The interpretation of the results is wide open at present.

If it turns out that the microwave radiation field departs strongly from the blackbody form, then either extra components are present, or the radiation has a quite different origin. Also, if we live in a steady-state universe the radiation must have another origin. In both cases the answer must be that the excess radiation, or the total flux, ultimately arises in discrete sources. A number of investigations have been made in which these possibilities have been explored. The sources are most likely to be the nuclei of galaxies which radiate largely at infrared and microwave frequencies. We shall return to the various theoretical possibilities later.

The discovery of the microwave background radiation by Penzias and Wilson in 1965 undoubtedly had a great impact on cosmology, possibly the greatest impact since Hubble and Humason firmly established, some forty years ago, that the universe is expanding. It was this latest discovery that led to the large number of theoretical investigations concerned with the early history of the universe. What should be made clear, however, is that the existence of this radiation is very powerful evidence for an initially dense state, provided that the radiation has a black-body spectrum as is predicted in the simple theory. If it turns out that it departs strongly from the blackbody shape, many attempts will undoubtedly be made to explain it in terms of a primordial radiation field plus large contributions from discrete sources, or even a more complex big bang. However, this must not obscure the important point that the absence of a black-body form would mean that the prediction has not been fulfilled and the strong and direct evidence for an initial dense state will have disappeared.

Another important aspect of this radiation is its isotropy. We shall discuss this in a separate section later.

Finally, it should be pointed out that this radiation is far more energetic than any of the other radiation fields known to exist.

\section{B. STARLIGHT}

The fact that large numbers of galaxies are spread fairly uniformly throughout the universe means that there must be an intergalactic radiation field due to the stars in these galaxies. Attempts to measure it are very difficult because to obtain an estimate of the extragalactic component of the night sky, it is necessary to subtract the Galactic component and the zodiacal light. The attempts made by Roach and Smith have already been mentioned. A number of theoretical attempts have been made to estimate the energy density of this radiation. Probably the best estimate is that by Felten (1966) who found that $\varrho_{s t}=1.5 \times 10^{-14} \mathrm{erg} \mathrm{cm}^{-3}$. He pointed out that this value is not very sensitive to the cosmological model which is assumed.

\section{RADIO BACKGROUND RADIATION}

The isotropic component of non-thermal radio emission has a maximum brightness temperature of $65 \pm 5 \mathrm{~K}$ at $178 \mathrm{MHz}$ (Turtle and Baldwin, 1962; Bridle, 1967). 
Measurements at a number of frequencies give a spectral shape which suggests that it is the result of the integrated effect of discrete sources. These are of two types, the strong radio sources, and the so-called normal galaxies. Bridle has argued that this isotropic component is largely due to the strong radio sources. He believes that the normal galaxies cannot make a significant contribution. It has been argued by the Cambridge group that the background radiation is generated by such strong sources which have gone through the density evolution which is invoked by that group to explain the counts of radio sources. On the other hand, Brecher and Morrison (1969) have concluded that normal galaxies - weak radio sources - contribute a large part of the background. The differences between these two interpretations appears to depend on what assumptions are made about the luminosity function of radio galaxies and its extrapolation at the faint end.

\section{X-RAY BACKGROUND RADIATION}

We have already mentioned that there is some evidence for an isotropic flux of soft $\mathrm{X}$-rays which may be due to bremsstrahlung from a low-density intergalactic plasma. However, apart from this, there is strong evidence for an isotropic background X-ray flux in the energy range $\sim 1 \mathrm{keV}$ to $\sim 100 \mathrm{MeV}$. It is of power law form $I\left(E_{x}\right) \propto E_{x}^{-\alpha}$ where $\alpha \simeq 2$ over the entire range. If the observational data are taken literally $\alpha \simeq 1.5$ for $1 \mathrm{keV} \leqslant E_{x} \leqslant 40$ and $\alpha \simeq 2.3$ for $40 \mathrm{keV} \leqslant E_{x} \leqslant 1 \mathrm{MeV}$. The energy density of this radiation amounts to $\sim 10^{-16} \mathrm{erg} \mathrm{cm}^{-3}$. A large number of speculations have been made to explain this background radiation (cf. the critical review by Brecher and Burbidge, 1970). The main point that we wish to make here is that it is agreed by everyone that the sources must ultimately involve a population of discrete objects. Either it is argued that the background is made up of the integrated radiation from a population of discrete sources, galaxies of some type which generate X-rays, or else it is supposed that discrete sources - radio sources, Seyfert galaxies, or some other classes of objects, eject high energy particles which interact with the microwave background radiation and/or intergalactic gas to give rise to the background flux.

\section{The Isotropy of the Microwave and the X-Ray Background Radiation Fields and Their Relation to Possible Theories Concerning Their Generation}

Both the microwave background and the X-ray background have been shown to be highly isotropic. From the measurements of isotropy that have been made, a number of deductions have been made concerning the conditions under which the radiation has been produced. We discuss the radiation fields in turn.

\section{A. ISOTROPY OF THE MICROWAVE BACKGROUND}

It has been shown that the microwave radiation is highly isotropic on the large scale to about $0.1 \%$ assuming that the radiation is blackbody radiation at $3 \mathrm{~K}$ (Partridge and Wilkinson, 1967), and on the small scale so that it is structureless to less than $0.2 \%$ on an angular scale of $1^{\circ}$ (Conklin and Bracewell, 1967). These results have been used as arguments in favor of the view that it arose in a big bang, and theoretical 
studies have been made of its consequences for the physics of the early universe. If it is relict radiation then its isotropy immediately tells us something about the universe at the epoch when the radiation was last scattered. If we designate $z_{0}$ as the redshift corresponding to unit optical depth for Thomson scattering, we find that, if the intergalactic density of ionized gas is $\varrho_{c}, z_{0} \simeq 7$. However, we have shown in the earlier discussion that there is certainly no evidence that the intergalactic density of hot gas is as large as $\varrho_{\dot{c}}$. If the density is very much less than this, then we are looking back to values of $z_{0} \sim 10^{3}$ or so. Investigations of the implication of these results for the evolution of the early universe have been made by Thorne (1967), Novikov (1968), and James (1969).

Whatever the degree of isotropy of the background radiation at such an epoch, it is possible that small anisotropies may have developed because of the presence of density inhomogeneities in the paths of propagation of the photons to the observer, causing gravitational frequency shifts. These might be caused by clusters of galaxies and the like. This problem was investigated by Sachs and Wolfe (1967), Rees and Sciama (1968), and Wolfe (1969). The theoretical investigations were applied on the basis that there was some observational evidence that there was a departure in isotropy $\delta T / T \simeq 0.5 \%$ (Partridge and Wilkinson, 1967) and that the direction in which this is seen might coincide with a distant density fluctuation, namely a cluster of QSOs. In fact there is apparently now doubt whether such an anisotropy is present. At the same time the clustering properties of QSOs are also in doubt. No proper analysis of their distribution on the sky has been carried out since the early work of Strittmatter et al. (1966), work which has been unfairly criticized by some, though by now many more redshifts are known. Of course there is still no certainty that QSOs lie at cosmological distances. Thus, at present it must be said that these theoretical investigations have so far told us little.

The existence of this highly isotropic radiation field means that it can be used as a reference system against which the observer can measure his velocity and direction of motion. We shall, therefore, be measuring the vector sum of the Earth's velocity about the Sun, the Sun's velocity around the Galaxy, and the velocity of the Galaxy in the local group or the supercluster. Since all of these components, with the exception of the first, are of the order of several hundred $\mathrm{km} \mathrm{s}^{-1}$, we should expect to see an anisotropy in the apparent temperature corresponding to a total velocity vector with a magnitude of $\sim 300 \mathrm{~km} \mathrm{~s}^{-1}$ or less. The variations in temperature are measured by observing as the earth rotates, so that the Earth's peculiar velocity shows up as a 24-hr anisotropy. Since there is no $24-\mathrm{hr}$ anisotropy to $0.1 \%$, we can put a limit on the peculiar velocity $v_{\text {total }}<300 \mathrm{~km} \mathrm{~s}^{-1}$ at a declination $\delta$ of $-8^{\circ}$ (the declination of the observations of Partridge and Wilkinson). Sciama (1967) and earlier Stewart and Sciama (1967) have carried out careful studies of the velocity components associated with the rotation of the Galaxy, motion of the Galaxy in the Local Group, and in the Virgo supercluster, and found that the net peculiar velocity should be about $300 \mathrm{~km} \mathrm{~s}^{-1}$. More recently Conklin (1969) claimed to have found a 24-hr anisitropy which he concluded corresponded to a peculiar velocity of $160 \mathrm{~km} \mathrm{~s}^{-1}$. This value 
has been re-estimated by Sciama (1969) to be about $210 \pm 100 \mathrm{~km} \mathrm{~s}^{-1}$. At this stage these results cannot be considered to be conclusive. Everyone is aware that, at some level, an anisotropy should be found, but it is clear that the observations and the interpretations are at the borderline of what is expected.

We turn now to a brief discussion of alternative explanations of the microwave background radiation. These are of great importance because:

(a) It is certainly not yet clear whether or not the microwave background has a black-body form;

(b) We may live in a steady-state universe in which case there was no primeval fireball.

The alternative models require that a large population of discrete sources gives rise to the observed spectrum. Many investigations of this type have been made, the most detailed being that of Wolfe and Burbidge (1969). These sources must radiate powerfully in the millimeter and submillimeter wave bands, and they must have weak radio components. It is now known that Seyfert galaxies and QSOs have some of these characteristics, and until surveys in the infrared and millimeter wavelength bands have been made we shall not know from direct observation what the situation is. However, those who believe strongly in the primeval fireball origin have argued that the high degree of isotropy argues against this type of explanation. It is true that the small scale isotropy measurements of Conklin and Bracewell require that, in all models, the space density of such discrete sources be comparable with, and in the case of the steady-state universe, much greater than the space density of ordinary galaxies (Hazard and Salpeter, 1969; Penzias et al., 1969). Whether or not this is a powerful argument against the hypothesis depends on one's point of view. Such a large population of intrinsically weak objects probably means that it will be very difficult to detect them as individual sources. At the same time it may well be that there is a large population of objects much less massive than ordinary galaxies, and we also know now that non-thermal emission in these previously unexplored parts of the electromagnetic spectrum is much more important than anyone had ever thought.

Another possible approach which will undoubtedly be explored is to argue that the observed microwave background consists of two components;

(i) a black body spectrum from a primeval fireball; and

(ii) a discrete source component.

Already, Setti and Woltjer (1970) have made such a proposal. They have attempted to explain the high flux in the $0.1-1 \mathrm{~mm}$ wavelength range as due to the integrated effect of Seyfert nuclei. They argue that the frequency of Seyfert nuclei may be an increasing function of $z$, going as $(1+z)^{6}$ up to $z \simeq 2.4$ as has been derived for cosmologically distant QSOs by Schmidt. Of course the procedure is rather arbitrary. If one allowed a larger fraction of galaxies to evolve in this way one could easily obtain too much energy in this wavelength band.

B. ISOTROPY OF THE X-RAY BACKGROUND

In the case of the X-ray background (measured at energies $\sim 10 \mathrm{keV}$ ) it has been 
found by Schwarz (1970), after averaging over all of the X-ray data from the OSO III satellite (which covers $\sim 50 \%$ of the sky) that there are no rms intensity fluctuations $(\delta I / I)_{\text {observed }}$ more than $3 \%$. Also, Schwarz has shown that the upper limits on any $12-\mathrm{hr}$ or 24-hr variations are less than $1 \%$. Wolfe (1970) has shown that these latter results set limits on the large scale shear and vorticity for approximately half of the sky. Because of a global analogy to the Compton-Getting effect the limiting values for the shear and the vorticity are the same as those set by the isotropy of the microwave background if intergalactic hydrogen now exists at densities $>10^{-30} \mathrm{~g} \mathrm{~cm}^{-3}$ for the shear model and $10^{-31}$ for the vorticity model. If there is very much less intergalactic gas than this, the limits are not as significant as those implied by the microwaves, but they are still important because they cover a much larger solid angle. The reason why these limits can be set by measuring isotropy limits on a flux which is in some way generated through discrete sources is that Wolfe has assumed, as have many others, that the bulk of the X-ray emission has been generated at early epochs, ranging from $z \sim 2$ to $z \sim 100$, depending on the model. These models are frequently used but are highly arbitrary.

A different approach is to ask whether the isotropy of the X-rays is compatible with the lumpy distribution of galaxies on the assumption that the bulk of the X-rays are produced at redshifts $<1$, i.e. the luminosity of the sources does not change with time. This problem has recently been investigated by Wolfe and Burbidge (1970). If it is assumed that the sources which generate $\mathrm{X}$-rays directly, or which generate electrons which make X-rays by Compton scattering in intergalactic space, follow the distribution of galaxies, i.e. the sources are arranged in lumpy structures characterized by length scales and density excesses corresponding to clusters or superclusters, then the expected fluctuation level exceeds the level observed. Various explanations for this result can be suggested.

To some it may be an argument in favor of the idea that the bulk of the X-rays were generated at much earlier epochs when it must be argued that the distribution of matter was much smoother. Alternatively, an attractive possibility is that this is evidence that the X-rays were generated in low luminosity sources which are distributed more smoothly than the bright galaxies. These sources in their numbers and power levels are similar to those required to explain the microwave background if one takes the discrete source model approach.

If the X-rays are generated largely by Compton scattering in intergalactic space, it may be asked whether a lumpy source distribution may not be smoothed out because the Compton scattering length for electrons to produce $10 \mathrm{keV}$ photons is about $50 \mathrm{Mpc}$. However, to smooth out irregularities it would be required that the scattering length is very large compared with the separations between clusters or superclusters, and this is not the case. In addition, it does not appear that the electrons can freely escape from galaxies and travel directly $\sim 50 \mathrm{Mpc}$ before undergoing a Compton scattering. They are likely to propagate in such a way that the linear distance they travel from the source is very much less than the scattering length. 


\section{Theoretical Studies of Diffuse Intergalactic Matter}

If we wish to investigate theoretically the properties of matter concerning which there is very little or no observational evidence, it is necessary first to make some assumptions about cosmology. Nearly all investigators in this field have taken the view that we live in an evolving universe and have attempted to work out the consequences of such a model for the evolution of intergalactic gas. There are a few who either feel that they do not know whether this model is correct or not, or adhere to the steady-state theory. Thus they have not felt it worthwhile to study the evolution of intergalactic gas in an evolving universe. And then there are those who have no strong cosmological prejudices but who believe that other types of observations, and in particular, studies of the galaxies and galactic nuclei, suggest that, independent of cosmology, the key to our understanding of what lies in the space between the galaxies is in the galactic nuclei themselves. The basic theoretical ideas are therefore:

(1) That the universe started in a primeval fireball and that the intergalactic matter is that left over after galaxies formed at an early stage in the expansion;

(2) That the universe started in a highly condensed state, but that galaxies formed out of high density configurations left behind in the expansion - the so-called delayed cores. It is not clear in this type of model what fraction of the matter will remain in the form of diffuse gas. However, the observational evidence shows unequivocally (cf. Burbidge, 1970) that matter is continuously being ejected from galactic nuclei and this will indeed make a contribution to the intergalactic medium;

(3) That we live in a steady-state universe in which matter is being continuously created in the nuclei of galaxies. If this is the case, the only diffuse intergalactic material will be that which is ejected from galactic nuclei.

Nearly all of the theoretical investigations have been directed toward (1) as follows:

It is easy to calculate the way in which the density and temperature of matter and radiation vary with the scale factor $R$ as the universe expands from a big bang. At temperatures greater than $\sim 10^{9} \mathrm{~K}\left(T_{1} \simeq m_{e} c^{2} / k\right)$ the radiation is in equilibrium with a thermal gas of nucleons and relativistic electrons and positrons. Using the first law of thermodynamics, it is easy to show that for $T>T_{1}, T_{\text {matter }}=T_{\text {radiation }} \propto R^{-1}$, and $\varrho_{\text {matter }}=\varrho_{\text {radiation }} \propto R^{-4}$. As the temperature falls below $T_{1}$, radiation and electron positron pairs are no longer in equilibrium and the pairs annihilate and feed their rest energy into the radiation field. The matter density drops sharply, but since the gas is hot and ionized, it is strongly coupled to the radiation field by atomic interactions - photoionization, radiative recombination, Compton scattering and bremsstrahlung, and $T_{\text {matter }}=T_{\text {radiation }} \propto R^{-1}$. Since the number of particles is constant, $\varrho_{\text {matter }} \propto R^{-3}, \varrho_{\text {radiation }} \propto R^{-4}$. As the gas and the radiation cool, they remain in thermal equilibrium until the gas recombines, at which point they uncouple and evolve separately, so that $T_{\text {matter }} \neq T_{\text {radiation. }}$. For the uncoupled components $T_{\text {radiation }} \propto R^{-1}$, $\varrho_{\text {radiation }} \propto R^{-4}$, while $T_{\text {matter }} \propto R^{-2}, \varrho_{\text {matter }} \propto R^{-3}$.

If one now assumes that the microwave radiation is black body radiation generated by the big bang and that $T_{\text {(radiation now) }}=3 \mathrm{~K}$ and one also assumes a value for the 
mean gas density now, then the temperature and epoch at which the radiation and the matter uncoupled can be determined. If the intergalactic density has the critical value $\varrho_{c}$ (and in our previous discussion we showed that there was really no evidence for this), then it is found that the decoupling took place when $R=10^{-3} R_{\text {present }}$ or at a redshift of about 1000 and that the temperature was about $4000 \mathrm{~K}$ ( $50 \%$ ionization). Since the dependence of $\varrho$ on $R$ is very steep, the values will not change very much for $\varrho \ll \varrho_{c}$ and close to the value obtained for galaxies alone.

Despite the uncertainties discussed earlier, theoreticians working in this field have then almost without exception chosen to believe that the absence of Ly- $\alpha$ in absorption in the spectra of QSOs means that the gas must be highly ionized, rather than that the QSOs are close by. They have also chosen to believe that the soft X-ray flux is coming from this hot intergalactic gas at critical density. So the problem has then been to calculate what is responsible for the re-heating of gas which otherwise would be exceedingly cool at epochs later than $z \sim 1000$. It has been argued that the heating is due to high-energy charged particles (cosmic rays) generated in an epoch of galaxy formation somewhere in the range $z=2-10$. This epoch has been chosen again because theoreticians have chosen to interpret the redshifts of QSOs as cosmological, and thus their distribution in redshifts as indicating a number density $N$ proportional to $(1+z)^{6}$ with a flattening in slope beyond $z \simeq 2.4$ (Schmidt and others), and also because they accept the Cambridge interpretation of the $\log N-\log S$ curve as indicating density or luminosity evolution in the radio sources at epochs in the range $z \sim 2-10$ (cf. Longair, 1966, 1970). The calculation of the atomic processes involved in the heating and cooling of this gas has been carried out by many people including Weymann (1967), Ginzburg and Ozernoy (1966), Gould and Ramsay (1966) and most recently Bergeron $(1969,1970)$. Calculated models can be made to fit these interpretations of the observational data.

On these assumptions, it has also been conjectured by some that galaxy formation is related to the high density of radio sources and QSOs at this epoch. The problems of galaxy formation lie outside the scope of this lecture. However, the conventional approaches were recently extensively reviewed by Field (1970). With the great interest in the evolving universe models there have been a number of papers in this field very recently, including those of Peebles and Dicke (1968), Doroshkevich et al. (1967), Ozernoy and Chernin (1968, 1969), and most recently, Oort (1970). As has been stressed elsewhere, these ideas require that one has to postulate the existence of initial density fluctuations which cannot be statistical, but must come from highly non-linear effects or the properties of matter and radiation at exceedingly high densities in a very early universe. In this sense the theory is very ad hoc. Oort and Ozernoy and Chernin have appealed to primordial turbulence as originally enunciated by von Weizsäcker to explain both the density fluctuations, and the angular momenta of galaxies. Oort has argued that the protogalaxies initially expand with the universe and only collapse at much later times $\left(t \geqslant 10^{9} \mathrm{yr}\right.$ ago $)$. He has speculated that this age of collapse may correspond to the birth of radio sources, i.e., to the period $z \simeq 2-10$. $\mathrm{He}$ has also argued on dynamical grounds that the bulk of the primeval gas will not 
be condensed into galaxies, and that the ratio of uncondensed matter to that in galaxies means that the mass-energy density in the universe is close to the critical value $\varrho_{c}$.

\section{High-Energy Charged Particles in Intergalactic Space}

The existence of strong radio sources shows that large fluxes of relativistic particles are ejected from galaxies. While we do not know to what extent the electrons are depleted in the radio sources themselves, there is every reason to argue that a flux of protons and heavier nuclei will be generated with them, and throughout most of the energy range associated with cosmic rays (from about $100 \mathrm{MeV}$ per nucleon to $10^{18} \mathrm{eV}$ per nucleon) they will not experience appreciable energy losses by interactions with matter or radiation in the sources. Thus, it can be assumed that radio sources inject cosmic rays into intergalactic space. However, there is no agreement as to how large an energy density of intergalactic cosmic rays is likely to be present due to such processes. Because of the problems associated with the acceleration and trapping of cosmic rays in our Galaxy, most have argued that the highest energy $\left(>\sim 10^{17} \mathrm{eV}\right)$ cosmic rays are likely to have an extragalactic origin. However, the most recent fashion has been to attribute galactic cosmic rays to acceleration near the surface of pulsars and some have claimed that acceleration of the whole cosmic ray spectrum by pulsars is possible, though no convincing demonstration of the mechanism which does this has yet been given. If only the highest energy cosmic rays have an extragalactic origin their energy density is exceedingly small $\left(\approx 10^{-16} \mathrm{erg} \mathrm{cm}^{-3}\right)$.

However, if there are very high energy cosmic rays in intergalactic space, they may be photodisintegrated by photons of the microwave background at an energy threshold given by $E_{c} \simeq\left(m_{\pi} c^{2}\right)^{2} / h v$ where $m_{\pi}$ is the mass of the pion and $h v=k T$ where $T$ is the blackbody temperature of the microwave background. For $T \simeq 3 \mathrm{~K}, E_{c} \simeq 10^{18} \mathrm{eV}$. Greisen (1966) and others have made detailed calculations of this effect and have attempted to see whether there is any attenuation of the primary cosmic ray spectrum which can be attributed to their destruction by microwave photons. The observational situation is complicated because the expected flux of cosmic rays with energies above $\sim 10^{18} \mathrm{eV}$ is very small. Even if it is eventually found that there is no progressive cutoff in the primary cosmic ray spectrum due to this effect, there will be no need to argue, as some have already done, that the radiation field does not pervade the whole of space. It must simply be concluded that the very highest energy cosmic rays are generated at distances less than a mean free path for nucleon-photon collisions (1-10 Mpc for a microwave background with $T \approx 3 \mathrm{~K}$ ). If the microwave radiation is more intense and energetic than this, the originating volume for the cosmic rays will be cut down further.

The total energy density of cosmic rays between the galaxies is not known. The number that one takes depends on one's ideas concerning the origin of cosmic rays. Most people at present have accepted the supernova theory of Ginzburg and Syrovatsky as they have developed and modified it over the years, taking into account the possibility that a significant fraction of the galactic cosmic rays could be generated in 
the galactic center. In this galactic theory the leakage from the Galaxy must be kept rather small, and the intergalactic cosmic ray energy density which arises from leakage from radio galaxies and ordinary galaxies is always quoted by these authors to be $\approx 10^{-14} \mathrm{erg} \mathrm{cm}^{-3}$. There is certainly no question but that the intergalactic high-energy electron density must be very small, as electrons are rapidly destroyed by microwave photons in Compton collisions. On the other hand, Burbidge and Hoyle have proposed that cosmic rays, largely nucleons, are universal, and the intergalactic energy density is equal to that at the top of the Earth's atmosphere, namely about $10^{-12} \mathrm{erg} \mathrm{cm}^{-3}$. If this theory is correct cosmic rays are generated in a wide variety of nuclei of galaxies and QSOs and the energy density is comparable with that of the microwave background which may also have a discrete source origin. One remarkable feature of the primary cosmic rays is that they, along with the microwaves and the X-rays, show a remarkably high degree of isotropy (Elliot, 1969). If they do not have an extragalactic origin, the galaxy being 'open', then to explain this isotropy causes some difficulties, and in particular it must be argued that the cosmic rays which we detect have been generated in a very 'local' region in a spiral arm.

Intergalactic cosmic rays, if there are any, will interact with intergalactic gas, if there is any. The most conspicuous product of this interaction will be $\gamma$-rays from the decay of neutral pions produced in proton-proton collisions. Since there is a marginally detected flux of $\gamma$-rays which may have come from $\pi_{0}$ decay (Clark et al., 1968) calculations have been made (Gould, 1967; Ginzburg, 1969; Stecher, 1969; Cavallo, 1970) which enable us to check whether or not a universal cosmic ray flux and an intergalactic gas at critical density can co-exist, and not violate the upper limits on the $\gamma$-ray flux observed. The answer appears to be, marginally, no. However, as was discussed earlier, there is really no evidence for the presence of gas, hot or cold, at critical density, so this is not evidence against the universal cosmic ray hypothesis.

\section{Neutrinos and Antineutrinos in Intergalactic Space}

Because neutrinos interact so weakly with matter they are exceedingly difficult to detect. The only neutrinos of cosmic origin which have certainly been detected are the few from the Sun found by Davis. However, there have been some suggestions that neutrinos may be present in sufficient numbers so that they make a significant contribution to the total mass-energy density in the universe.

Pontecorvo and Smorodinsky (1962) and Weinberg (1962) suggested that there may exist a background neutrino flux with low energy but perhaps with enough energy to give the critical mass density. If one assumes that a gas of neutrinos or antineutrinos has a distribution in phase space corresponding to the lowest possible mean energy, then we are dealing with a completely degenerate Fermi gas. By normal methods one then finds that the energy density

$$
\varrho_{v}=1.64 \times 10^{12} E_{F}^{4} \mathrm{eV} \mathrm{cm}^{-3} .
$$

For a Friedman model the critical density, when neutrinos or radiation are dominant, is given by $q_{0} \geqslant 1$, so that $\varrho_{v}($ critical $)=\varrho_{c}=1.1 \times 10^{-29} \mathrm{erg} \mathrm{cm}^{-3}$. 
If this neutrino sea is present at this density, we find that

$$
E_{F} \simeq 0.01 \mathrm{eV}
$$

Now the beta-decay spectrum will be cut off at an electron energy below the maximum energy available, if there is a sea of antineutrinos, since in the reaction

$$
n \rightarrow p+e+\bar{v}_{e}
$$

the final antineutrino cannot have an energy lower than $E_{F}$ for the antineutrino gas.

An experimental upper limit for $E_{F}$ for antineutrinos has been obtained from studies of the upper end of the beta-decay spectrum of $T^{3}$ and it is found that (Bergkvist, 1969)

$$
E_{F}<60 \mathrm{eV}
$$

or a factor of 6000 above $E_{F}$ corresponding to $2 \varrho_{c}$. Since $\varrho_{\nu} \propto E_{F}^{4}$, it is clear that this experiment is very far from setting meaningful limits to the energy density of low energy neutrinos in the universe.

Other limits can be set by interactions of high energy cosmic rays with the hypothetical sea of neutrinos and/or antineutrinos. There are uncertainties about the cross sections, the form of the cosmic ray spectrum at high energies and the question whether a cutoff is even due to neutrinos, rather than to microwave photons. For some models one can obtain limits to the Fermi energy $E_{F}$ much lower than that obtained from the beta decay spectrum, but still high enough only to set limits to $\varrho_{v}$ which are far above the critical density.

Rather than assume that there is present a large neutrino flux and try to set limits to its energy density, one can ask what physical processes may have given rise to neutrinos and how plentiful will they be.

If one accepts a big bang model with a primeval fireball, there will now be a blackbody energy distribution of neutrinos which is related to the blackbody photon distribution. It has been shown (de Graaf, 1969) that at the decoupling phase

$$
T_{v}=\left(\frac{4}{11}\right)^{1 / 3} T_{\gamma}
$$

Since both neutrino and photon distribution cool in the same way, if $T_{\gamma}$ now $\approx 3 \mathrm{~K}$, $T_{v} \approx 2 \mathrm{~K}$, so that the energy density is $\sim\left(\frac{2}{3}\right)^{4}$ or about $20 \%$ of the photon energy density, i.e. it is negligible as far as a contribution to the critical mass is concerned.

Another way of estimating the possible neutrino flux is to try to calculate how much energy is emitted in the form of neutrinos in stellar evolution. De Graaf (1969) has recently done this, using the work of Arnett on the final stages of evolution of stars with masses $\sim 10-30 M_{\odot}$ which are likely to be most prolific in neutrino production. He finds an upper limit $\sim 3 \mathrm{eV} \mathrm{cm}^{-3}$ for the energy density, again a very low value as compared with $\varrho_{c}$. Estimates of the total neutrino production by main sequence stars in all galaxies over $10^{10} \mathrm{yr}$ give an energy density of $10^{44} \times 10^{9} \times 10^{17} \times 3 \times$ $\times 10^{-85} \times 10^{-2} \approx 3 \times 10^{-17} \mathrm{erg} \mathrm{cm}^{-3}$, or $\sim 10^{-5} \mathrm{eV} \mathrm{cm}^{-3}$. 


\section{Gravitational Radiation}

Recently Weber $(1969,1970)$ has claimed to have detected gravitational waves which, if real, are likely to have been emitted in processes of unknown origin from the center of our own Galaxy.

The results are remarkable because, if correct, they indicate that our Galaxy is currently radiating gravitational energy at a rate of $10-1000 M_{\odot} \mathrm{yr}^{-1}$. This means that, if the radiation from a typical galaxy is of this order, and has continued for $\sim 10^{10} \mathrm{yr}$, mass comparable to, or greater than, the mass of the galaxy will have been radiated. Consequently, it must be concluded that the majority of the mass-energy in the universe is currently present in the form of gravitational radiation. However, these ideas are based on such tenuous observational data that little weight can be given to them at present.

If there was a hot big bang, there will be some gravitational radiation of blackbody form present today. The temperature of this radiation has been estimated by Weinberg to be about $1 \mathrm{~K}$.

\section{Conclusion}

In this paper I have attempted to discuss critically our present knowledge of intergalactic matter and radiation as it relates to cosmology. The observational evidence obtained so far should be looked at from two different points of view as far as cosmological models are concerned.

The most commonly advocated model is one in which the universe started in a hot big bang and galaxies or protogalaxies were formed from large density fluctuations that were already present at a time very close to $t=0$. In such a model we might expect that a considerable amount of primeval gas has never condensed into galaxies, and is spread throughout the space between the galaxies. It is not at all clear what fraction of the matter would also be present in the form of objects with very high mass-to-light ratios - either galaxies which have never formed stars, or galaxies in which the stars have evolved to their final evolutionary stages. The microwave background radiation in this model is relict radiation, while the other electromagnetic radiation fields arise directly (starlight, non-thermal radio emission, X-rays), or indirectly (X-rays) in discrete objects which have formed from the initial lumps which have a cosmological origin.

The alternative picture is one in which we live in a steady state universe, or an evolving universe in which galaxies form from matter which is ejected from 'prestellar' high density configurations which are primeval (Ambartsumian), or in which matter is created in galactic nuclei and the like (Hoyle and Narlikar; McCrea).

Since there is no certain evidence for any significant amounts of intergalactic matter at all, we are still at liberty to interpret the largely null observations according to either picture. The data so far available tell us effectively nothing about the value of $q_{0}$, or whether or not $\Lambda=0$ in the Friedman models. On the astrophysical side the idea that there is a considerable amount of uncondensed matter is not ruled out. 
On the other hand, the limits set do not conflict with the view that whatever diffuse matter is present has been ejected from galaxies. In the steady state and delayed core models we would expect that all of the diffuse intergalactic matter has been ejected from nuclei. Large numbers of dark discrete objects might exist and be compatible with either picture.

In a steady-state universe the microwave radiation must be generated in discrete sources. As has been pointed out earlier, the complications in the observed data are such that it can no longer be asserted with any certainty that the radiation is relict, nor is there any good evidence in favor of the view that it must have come from discrete sources.

Thus, while there have been some observational successes and much theoretical speculation, studies of intergalactic matter and radiation have still not allowed us to draw certain conclusions about the origin and evolution of the universe.

\section{Acknowledgement}

Research in extragalactic astronomy at UCSD is supported by the National Science Foundation and by NASA under Grant NGL 05-005-004.

\section{References}

Abell, G. O.: 1965, Ann. Rev. Astron. Astrophys. 3, 2.

Allen, R. J.: 1969, Astron. Astrophys. 3, 316, 382.

Arp, H. C.: 1966, in Atlas of Peculiar Galaxies, California Institute of Technology, Pasadena.

Arp, H. C. and Bertola, F.: 1969, Astrophys. Letters 4, 23.

Bahcall, J. N. and Peebles, P. J. E.: 1969, Astrophys. J. Letters 156, L7.

Bahcall, J. N. and Salpeter, E. E.: 1966, Astrophys. J. 144, 847.

Bahcall, J. N. and Spitzer, L.: 1969, Astrophys. J. Letters 156, L63.

Baum, W. A.: 1955, Publ. Astron. Soc. Pacific 67, 328.

Bergeron, J.: 1969, Astron. Astrophys. 3, 42.

Bergeron, J.: 1970, Astron. Astrophys. 4, 335.

Bergkvist, K. E.: 1969, CERN Report 69-7, p. 91.

Bortolot, V. J., Clauser, J. F., and Thaddeus, P.: 1969, Phys. Rev. Letters 21, 1460.

Bowyer, C. S., Field, G. B., and Mack, J. E.: 1968, Nature 217, 32.

Brecher, K. and Burbidge, G. R.: 1970, Comments Astrophys. Space Phys. $2,75$.

Brecher, K. and Morrison, P.: 1969, Phys. Rev. Letters 23, 802.

Bridle, A. H.: 1967, Monthly Notices Roy. Astron. Soc. 136, 219.

Bunner, A. N., Coleman, P. C., Kraushaar, W. L., McCammon, D., Palmieri, T. M., Shilepsiky, A., and Ulmer, M.: 1969, Nature 223, 1222.

Burbidge, E. M.: 1965, Ann. Astrophys. 28, 164.

Burbidge, E. M.: 1971, Pont. Acad. Scient. Scripta Varia 35, 121.

Burbidge, G. R.: 1970, Ann. Rev. Astron. Astrophys. 8, 369.

Burbidge, G. R. and Burbidge, E. M.: 1967, in Quasi-Stellar Objects, W. H. Freeman, San Francisco.

Burbidge, G. R. and Burbidge, E. M.: 1969a, Nature 222, 735.

Burbidge, G. R. and Burbidge, E. M.: 1969b, Nature 224, 21.

Burbidge, E. M. and Burbidge, G. R.: 1970, Stars and Stellar Systems 9, University of Chicago Press, Chicago, in press.

Burbidge, E. M., Lynds, C. R., and Burbidge, G. R.: 1966, Astrophys. J. 144, 447.

Cavallo, G.: 1970, Ph.D. Thesis, University of California, San Diego.

Clark, G. W., Garmire, G. P., and Kraushaar, W. L.: 1968, Astrophys. J. Letters 153, L203. 
Conklin, E. K.: 1969, Nature 222, 971.

Conklin, E. K. and Bracewell, R. N.: 1967, Phys. Rev. Letters 18, 614; Nature 216, 777.

Davies, R. D. and Jennison, R. C.: 1964, Monthly Notices Roy. Astron. Soc. 128, 123.

Doroshkevich, A. G., Zeldovich, Y. B., and Novikov, I. D.: 1967, Soviet Astron. 11, 233.

Elliot, H.: 1969, Proc. Int. Cosmic Ray Conference, Budapest.

Felten, J. E.: 1966, Astrophys. J. 144, 241.

Felten, J. E. and Bergeron, J.: 1969, Astrophys. Letters 4, 155.

Field, G. B.: 1962, Astrophys. J. 135, 684.

Field, G. B.: 1970, preprint.

Field, G. B. and Henry, R. C.: 1964, Astrophys. J. 140, 1002.

Ginzburg, V. L.: 1968, Astrophys. Space Sci. 1, 125.

Ginzburg, V. L. and Ozernoy, L. M.: 1966, Astr. Zh. 42, 934; Soviet Astron. 9, 726.

Gold, T. and Hoyle, F.: 1959, in R. Bracewell (ed.), Paris Symposium on Radio Astronomy,

Stanford University Press, Palo Alto, p. 583.

Goldstein, S.: 1963, Astrophys. J. 138, 978.

Gould, R. J.: 1967, Am. J. Phys. 35, 376.

Gould, R. J. and Burbidge, G. R.: 1963, Astrophys. J. 138, 969.

Gould, R. J. and Ramsay, W.: 1966, Astrophys. J. 144, 587.

Graaf, T. de: 1970, Astron. Astrophys. 5, 335.

Greisen, K.: 1966, Phys. Rev. Letters 16, 748.

Gunn, J. E. and Peterson, B. A.: 1965, Astrophys. J. 142, 1633.

Hazard, C. and Salpeter, E. E.: 1969, Astrophys. J. Letters 157, L89.

Henry, R. C., Fritz, G., Meekins, J. F., Friedman, H., and Byram, E. T.: 1968, Astrophys. J. Letters 153, L11.

Houck, J. R. and Harwit, M.: 1969, Astrophys. J. Letters 157, L245.

Hubble, E.: 1936, in Realm of the Nebulae, Oxford University Press.

Hulsbosch, A. N. M.: 1968, Bull. Astron. Inst. Neth. 20, 33.

Karachentsev, I. D.: 1966, Astrofizika 2, 307 (English translation: Astrophysics 3, 1959).

Koehler, J. A. and Robinson, B. J.: 1966, Astrophys. J. 146, 488; Astrophys. J. 146, 504.

Kurt, V. and Sunyaev, R.: 1967a, JETP Letters 5, 299.

Kurt, V. and Sunyaev, R.: 1967b, Cosmic Res. 5, 496.

Kurt, V. and Sunyaev, R.: 1970, in L. Houziaux and H. E. Butler (eds.), 'Ultraviolet Stellar Spectra and Related Ground-Based Observations', IAU Symp. 36, 341.

Longair, M. S. : 1966, Monthly Notices Roy. Astron. Soc. 133, 421.

Longair, M. S.: 1970, Monthly Notices Roy. Astron. Soc. 150, 155.

Lynds, C. R. and Wills, D.: 1970, Nature 226, 532.

Muelhner, D. and Weiss, R.: 1970, Phys. Rev. Letters 24, 742.

Neyman, J., Scott, E. L., and Shane, D.: 1952, Astrophys. J. 117, 92.

Oort, J. H.: 1958, in La Structure et l'Evolution de l'Universe, Stoops, Brussels, p. 163.

Oort, J. H.: 1966, Bull. Astron. Inst. Neth. 18, 421.

Oort, J. H.: 1969, Nature 224, 1158.

Oort, J. H.: 1970, Astron. Astrophys. 7, 381, 405.

Ozernoy, L. M. and Chernin, A. D.: 1968, Soviet Astron. 11, 907.

Ozernoy, L. M. and Chernin, A. D.: 1969, Soviet Astron. 12, 901.

Partridge, R. B. and Wilkinson, D. T.: 1967, Phys. Rev. Letters 18, 557.

Peebles, P. J. E. and Dicke, R. H.: 1968, Astrophys. J. 154, 891.

Peebles, P. J. E. and Partridge, R. B.: 1967, Astrophys. J. 148, 713.

Penzias, A. A. and Scott, E. H.: 1968, Astrophys. J. Lett. 153, L7.

Penzias, A. A. and Wilson, R. W.: 1969, Astrophys. J. 156, 799.

Penzias, A. A., Schraml, J., and Wilson, R. W.: 1969, Astrophys. J. Letters 157, L49.

Pontecorvo, B. and Smorodinsky, G.: 1962, Soviet Phys. JETP 14, 173.

Rees, M. J.: 1969, Astrophys. Letters 4, 61.

Rees, M. J.: 1970, Astrophys. J. Letters 160, L29.

Rees, M. J. and Sciama, D.: 1968, Nature 217, 511.

Roach, F. and Smith, L. L.: 1968, Geophys. J. Roy. Astron. Soc. 15, 227.

Rubin, V. C., Moore, S., and Bertiau, F. C.: 1967, Astron. J. 72, 59.

Sachs, R. and Wolfe, A. M.: 1967, Astrophys. J. 147, 73. 
Sandage, A. R. and Luyten, W. J.: 1969, Astrophys. J. 155, 913.

Sargent, W. L. W.: 1970, Astrophys. J. 160, 405.

Scheuer, P. A. G.: 1965, Nature 207, 963.

Schmidt, M.: 1965, Astrophys. J. 141, 1295.

Schwarz, D.: 1970, Astrophys. J. 162, 439.

Sciama, D. W.: 1967, Phys. Rev. Letters 18, 1065.

Sciama, D. W.: 1969, in Proc. Varenna Summer School on General Relativity, in press.

Setti, G. and Woltjer, L.: 1970, Nature 227, 586.

Shivanandan, K., Houck, J. R., and Harwit, M.: 1968, Phys. Rev. Letters 21, 1460.

Shklovsky, I. S.: 1964, Astron. Zh. 41, 801 (Soviet Astron. 8, 638, 1965).

Shklovsky, I. S.: 1969, Soviet Astron. 13, 734.

Silk, J.: 1970, Astrophys. J. 160, 793.

Stecher, F. W.: 1969, Astrophys. J. 157, 507.

Stewart, J. M. and Sciama, D. W.: 1967, Nature 216, 748.

Stockton, A. N. and Lynds, C. R.: 1966, Astrophys. J. 144, 451.

Strittmatter, P. A., Faulkner, J., and Walmsley, M.: 1966, Nature 212, 1441.

Sunyaev, R.: 1969, Astrophys. Letters 3, 33.

Thorne, K. S.: 1967, Astrophys. J. 148, 51.

Turtle, A. J. and Baldwin, J. E.: 1962, Monthly Notices Roy. Astron. Soc. 130, 379.

Vaucouleurs, G. de: 1969, Astrophys. Letters 4, 17.

Vaucouleurs, G. de and Vaucouleurs, A. de: 1970, Astrophys. Letters 5, 219.

Vorontsov-Velyaminov, B. A.: 1959, Atlas of Interacting Galaxies, Moscow.

Weber, J.: 1969, Phys. Rev. Letters 22, 1320.

Weber, J.: 1970, Phys. Rev. Letters 24, 276; Phys. Rev. Letters 25, 180.

Weinberg, S.: 1962, Phys. Rev. 128, 1457.

Weymann, R. J.: 1967, Astrophys. J. 147, 887.

Wilkinson, D. and Partridge, R. B.: 1967, Nature 215, 719.

Wolfe, A. M.: 1969, Astrophys. J. 156, 803.

Wolfe, A. M.: 1970, Astrophys. J. Letters 159, L61.

Wolfe, A. M. and Burbidge, G. R.: 1969, Astrophys. J. 156, 345.

Wolfe, A. M. and Burbidge, G. R.: 1970, Nature 228, 1170.

Yu, J. T. and Peebles, P. J. E.: 1969, Astrophys. J. 158, 103.

Zeldovich, Y. B. and Sunyaev, R. A.: 1969, Comments Astrophys. Space Phys. 1, 159.

Zwicky, F.: 1957, Morphological Astronomy, Springer, Berlin.

\section{Discussion}

Silk: Firstly, Oort has suggested that the infall of intergalactic matter to the Galaxy is responsible for the predominantly negative radial velocities of the high velocity clouds. It should be emphasized that this interpretation is not unique, and that the density of intergalactic matter derived in this manner should be regarded as no more than an upper limit. In particular, the high velocity clouds that are actually observed in 21-cm emission must be predominantly of galactic origin, even in Oort's interpretation. Secondly, the limit on ultra-violet radiation found by Kurt and Sunyaev may be too low by between one and two orders of magnitude, according to recent measurements by Barth reported at the Lunteren Symposium (1969).

Oort: It is true that gas falling into our galaxy would probably initially have velocities of the order of $500 \mathrm{~km} \mathrm{~s}^{-1}$. But it would initially have a very high temperature and would not become visible before it has collided with gas in the galactic halo. It is difficult to determine at what velocity this would happen, but according to calculations made in Leiden, $200 \mathrm{~km} \mathrm{~s}^{-1}$ appears a plausible value.

Wagoner: It should be pointed out that all the existing far infrared observations, if taken at face value, imply the existence of a narrow emission feature $(\Delta \lambda \leqslant 0.1 \lambda)$ at $\lambda=0.87 \mathrm{~mm}$ which contains 25 times as much energy flux as a $2.7 \mathrm{~K}$ blackbody background. 